\title{
GPPS-BJ-2019-0017
}

\section{INVESTIGATION OF FILM COOLING VORTICAL STRUCTURES BEHIND A CYLINDRICAL HOLE FED BY AN INTERNAL CROSSFLOW}

\author{
Mohamed Qenawy, Wenwu Zhou*, Di Peng, Yingzheng Liu \\ School of Mechanical Engineering, Shanghai Jiao Tong University \\ 800 Dongchuan Road, 200240 Shanghai, China \\ Email: zhouww@sjtu.edu.cn
}

\begin{abstract}
The film cooling vortical structure behind a cylindrical hole fed by internal crossflow was investigated by large eddy simulation (LES). The simulation was performed on a single simple cylindrical hole (i.e., a spacing of $3 D$, an entry length of $6.5 D$, and an injection angle of $35^{\circ}$ ). Two blowing ratios $(M=0.40$ and 0.80$)$ were simulated with a density ratio of 0.97 . The results were validated in comparison with the measured data. The vortical structures were identified by the Q-criterion coherent isosurface and their unsteady signatures were identified by the standard deviation (SD). The vortical structures were demonstrated to play the main role in cooling performance and its instability. The internal flow was significantly produced a vortex tube structure, which responsible for the shear vortex (Kelvin-Helmholtz instabilities) between the coolant and the mainstream at the hole exit. As a result, it forced the legs of the counter-rotating vortex pair (CRVP) to widely spread and enhance the coolant effectiveness downstream.
\end{abstract}

\section{INTRODUCTION}

Nowadays, increasing the operating temperature of the gas turbine has been required to achieve its high performance. Such temperature exceeded the blade melting temperature, and thus the cooling technique was introduced. Film cooling is the widest cooling technique applied, where the coolant is ejected from discrete holes to form a thin layer over the blade surface to protect it. However, film cooling is known as a jetin-cross-flow (JICF) problem, in which a complex threedimensional flow mixing occurs at the hole exit (Andreopoulos \& Rodi, 1984; Karagozian, 2014). It produces a large number of vortices, which forms their vortical coherent structures (Fric \& Roshko, 1994; Mahesh, 2011) that force the coolant to be unstable over the blade surface (Kohli \& Bogard, 2011). Consequently, the coolant surface may be partially or completely exposed to the mainstream hot gases, which lead to the blade failure with the duty operation of the gas turbine. Therefore, understanding the film cooling flow structure is highly desirable.

Recently, Acharya and Kanani (Acharya \& Kanani, 2017) summarized the film cooling structures behind the simple inclined cylindrical holes. It characterized by different vortical structures including counter-rotating vortex pair (CRVP), horseshoe vortex, Kelvin-Helmholtz (K-H) vortices, hairpin vortices, and wake vortices. These structures significantly changed with coolant to mainstream scaling parameters (i.e., density ratio (Eberly \& Thole, 2013) $\left(D R=\rho_{c} / \rho_{m}\right)$, velocity ratio (Dai et al., 2016) $(V R=$ $\left.U_{c} / U_{m}\right)$, and blowing ratio (Sakai et al., 2014) $(M=D R$. $V R)$ ) and the mainstream boundary layer (Zhong et al., 2016). The literatures have been successfully investigated those structures by considering vertical plenum internal flow. However, the coolant in the realistic gas turbine flows perpendicular to the mainstream along the blade axial direction in internal coolant passages. This configuration is known as internal crossflow (Gritsch et al., 2003; Peng \& Jiang, 2012a; Peng \& Jiang, 2012b; Saumweber \& Schulz, 2009). It significantly changed the jet exit profile, coolant trajectory, and coolant flow field. Furthermore, Peng and Jiang (Peng \& Jiang, 2012b) and Acharya and Leedom (Acharya \& Leedom, 2012) did large eddy simulation (LES) and found significant change in the instantaneous flow behaviour as well. Although these studies reported the asymmetric of the CRVP, they have not investigated, presented, or even deeply discussed those structures.

Consequently, the vortical structures associated with the flow field could change with the internal flow effects, and thus this work investigates this behavior. The main objective is to perform a three-dimensional compressible LES approach to provide a detailed evaluation of the identified unsteady flow structures. The simulation was performed on a physical problem taken from the experimental work of the authors (Qenawy et al., 2019). The numerical results were 
validated by the measured data in terms of cooling effectiveness and flow data. The effectiveness is defined as;

$$
\eta=\frac{T_{m}-T_{a w}}{T_{m}-T_{c}} .
$$

where $T_{m}, T_{c}$, and $T_{a w}$ are the mainstream, coolant and adiabatic wall temperatures, respectively. The vortical structure was identified by Q-criterion coherent isosurfaces and streamwise vorticity. The coolant fluctuation was identified by the standard deviation (SD). This investigation expected to be helpful for gas turbine designers.

\section{NUMERICAL SETUP AND VALIDATION}

Based on the previous studies (Acharya \& Leedom, 2012; Kalghatgi \& Acharya, 2014; Peng \& Jiang, 2012; Sakai et al., 2014; Zhong et al., 2016), LES can accurately capture the film cooling flow structures obtained by the experiment. Consequently, LES was conducted in order to investigate the vortical structures caused by the internal crossflow effects. The simulation was performed using computational fluid dynamics commercial code ANSYS FLUENT (v17.1).

\section{Physical problem}

The physical problem is an experimental work taken from the authors (Qenawy et al., 2019). It was achieved with fast-response pressure sensitive paint (PSP) technique. The geometrical design of the test model was chosen based on the previous studies, which was a single cylindrical row of holes with hole diameter $(D)$ of $4.0 \mathrm{~mm}$. The holes have a spacing of $3 D$, entry length of $6.5 \mathrm{D}$ and an injection angle of $35^{\circ}$ with respect to the mainstream. During the experiment, the air as a mainstream flow was supplied with a constant speed $\left(U_{m}\right)$ of $\approx 10 \mathrm{~m} / \mathrm{s}$ and turbulence intensity of $\approx 3 \%$. The incoming flow was fully developed turbulent flow with a boundary layer thickness $(\delta)$ of $\approx 2 D$. The Nitrogen as a coolant flow was discharged from crossflow channel which had a $6.25 \mathrm{D}$ wide, $3.75 D$ height, and $100 D$ length. The channel had an inlet velocity $\left(U_{c h}\right)$ of $4 \mathrm{~m} / \mathrm{s}$ and turbulence intensity of $5 \%$. The channel outlet was varied according to the required blowing ratios. Both, coolant and mainstream, were kept at the ambient temperature of $300 \mathrm{~K}$ to give a corresponding density ratio of 0.97 .

\section{Governing equations}

The governing equations for LES are obtained by filtering the time-dependent Navier-Stokes equations. For compressible flow, the density Favre filtering is applied for any quantity $(\varphi)$ (i.e. $\tilde{\varphi}=\bar{\rho} \varphi / \bar{\rho}$ ). This process filters out the eddies whose scales are smaller than the filter width or the grid spacing that used in the simulation. Therefore, the resulted equations govern the dynamics of the large eddies. Continuity equation:

$$
\frac{\partial \bar{\rho}}{\partial t}+\frac{\partial\left(\bar{\rho} \tilde{u}_{j}\right)}{\partial x_{j}}=0.0
$$

Momentum equation:

$$
\frac{\partial\left(\bar{\rho} \tilde{u}_{i}\right)}{\partial t}+\frac{\partial}{\partial x_{j}}\left(\bar{\rho} \tilde{u}_{i} \tilde{u}_{j}\right)=-\frac{\partial \bar{p}}{\partial x_{i}}+\frac{\partial \widetilde{\sigma}_{i j}}{\partial x_{j}}+\frac{\partial \tau_{i j}}{\partial x_{j}} .
$$

where $\tilde{\sigma}_{i j}$ is the shear stress tensor;

$$
\tilde{\sigma}_{i j}=\mu\left(\frac{\partial \tilde{u}_{i}}{\partial x_{j}}+\frac{\partial \tilde{u}_{j}}{\partial x_{i}}-\frac{2}{3} \frac{\partial \tilde{u}_{k}}{\partial x_{k}} \delta_{i j}\right)
$$

and $\tau_{i j}$ is the sub-grid scale stresses;

$$
\tau_{i j}=\bar{\rho}\left(\widetilde{u_{\mathrm{i}} u_{\mathrm{j}}}-\tilde{u}_{i} \tilde{u}_{j}\right)
$$

which split into its isotropic and deviatoric parts;

$$
\tau_{i j}=\underbrace{\tau_{i j}-\frac{1}{3} \tau_{k k} \delta_{i j}}_{\text {Deviatoric }}+\underbrace{\frac{1}{3} \tau_{k k} \delta_{i j}}_{\text {Isotropic }} .
$$

Energy equation:

$$
\frac{\partial(\bar{\rho} \widetilde{h})}{\partial t}+\frac{\partial\left(\bar{\rho} \widetilde{u}_{j} \widetilde{h}\right)}{\partial x_{j}}+\frac{\partial\left[\bar{\rho}\left(\widetilde{u_{j} h}-\widetilde{u}_{j} \widetilde{h}\right)\right]}{\partial x_{j}}=\frac{\partial}{\partial x_{j}}\left(\bar{\rho} \lambda \frac{\partial \widetilde{h}}{\partial x_{j}}\right) .
$$

\section{Geometry and boundary conditions}

The computational domain and the flow conditions (Figure 1) were established to match the experiment. The mainstream inlet was set as a velocity inlet boundary condition with a turbulent boundary layer profile of $\left(u / U_{m}\right)=(y / \delta)^{1 / 7}$. The boundary layer thickness $(\delta)$ was adjusted to agree with the experimental boundary layer profile at $X / D=-5$ while the mainstream velocity, $U_{m}$, was set as $10 \mathrm{~m} / \mathrm{s}$ which gives a corresponding Reynolds number $\left(R e_{m}=\rho_{m} U_{m} D / \mu_{m}\right)$ of 2660 based on the hole diameter $(D)$. The mainstream outlet was set as a zero-pressure outlet boundary condition. The channel inlet was set as a velocity inlet boundary condition with uniform velocity profile and an inlet velocity magnitude of $4 \mathrm{~m} / \mathrm{s}$ (corresponding channel velocity ratio $\left(V R_{c h}=U_{c h} / U_{m}\right)$ of 0.4 and channel Reynolds number $\left(R e_{c h}=\rho_{c h} U_{c h} D_{h} / \mu_{c h}\right)$ of 5000 based on the channel hydraulic diameter $\left(D_{h}\right)$ ). A pressure boundary condition was applied at the crossflow channel outlet, and varied to give the desired blowing ratios $(M)$. The coolant and the mainstream flows had a temperature of $300 \mathrm{~K}$ and $290 \mathrm{~K}$, respectively. As the domain is air, this gives a corresponding density ratio $(D R)$ of $\approx 0.97$. The mainstream top wall was set as a symmetrical boundary condition while the side walls were set as a periodic boundary condition. Adiabatic with no slip wall boundary condition was applied to all other walls. Under these conditions, two blowing ratios $(M=0.4$ and 0.8$)$ were simulated.

\section{Solver}

The simulation was performed using the segregated solver, which employs an implicit pressure-correction scheme. The governing equations, (mass, momentum, and energy), described above were solved transiently by LES. The deviatoric part of the sub-grid scale stress tensor in equation 6 is modelled using the compressible form of the dynamic sub-grid scale model (Germano et al., 1991) as:

$$
\tau_{i j}-\frac{1}{3} \tau_{k k} \delta_{i j}=2 C \bar{\rho} \Delta^{2}|\tilde{S}| \tilde{S}_{i j} .
$$

where $\Delta$ is a band of the filter, the coefficient $C$ is determined from $C_{s}=\sqrt{C}$, the Smagolinsky constant, $C_{s}$, is determined by the method proposed by Lilly (Lilly, 1992), and $S_{i j}$ is the rate-of-strain tensor which can be written as:

$$
\tilde{S}_{i j}=\frac{1}{2}\left(\frac{\partial \widetilde{u}_{i}}{\partial x_{j}}+\frac{\partial \widetilde{u}_{j}}{\partial x_{i}}\right) .
$$


The sub-grid scale heat flux term in equation 7 is modelled by the same manner as:

$$
\bar{\rho}\left(\widetilde{u_{\mathrm{j}} h}-\tilde{u}_{j} \tilde{h}\right)=-\bar{\rho} \frac{C \Delta^{2}}{P r_{t}}|\tilde{S}|\left(\frac{\partial \widetilde{h}}{\partial x_{j}}\right) .
$$

The numerical domain (Figure 1b) was set as a fluid domain. The working fluid was set as an ideal gas with Sutherland formula for viscosity, and kinetic theory for thermal conductivity. The spectral synthesizer method was applied on the mainstream and the coolant incoming flow conditions to generate a time-dependent, perturbed inlet condition with 3\% and 5\% turbulence intensity for the mainstream and the coolant flow, respectively.

The pressure and velocity were coupled using the SIMPLEC Scheme. Least squares cell-based method was used for the gradient discretization. The pressure term was discretised by second-order scheme. The diffusion terms were discretized using second-order upwind scheme. The convective terms were discretized using central-difference scheme. The time was discretized using bounded secondorder implicit scheme. The physical time step $(\Delta t=$ $\left.0.025 \mathrm{D} / U_{m}=1 e-5\right)$ was used with $20 \mathrm{sub}$-iteration for each time step. The Courant-Fredrichs-Levy $(C F L=$ $\left.U_{m} \Delta t / \Delta X\right)$ criterion was less than 0.64 . The simulation was run for 20 flow-through time (time required to pass through the whole domain $\left.(25 D), \Delta T=L / U_{m}\right)$ to convert all the transients out of the domain. For all the relative residuals, the convergence criteria were below $10^{-4}$. Flow statistics were then collected to obtain statistically steady state results for next 10 flow-times.

\section{Numerical validation}

A high-quality hexahedral mesh was performed using Star-CCM+ with special refinement of the near hole region. Most cells near the protected wall were refined to maintain $X^{+}$of $15, Y^{+}$of 0.2 , and $Z^{+}$of 15 based on the mainstream velocity. It is clearly seen in figure 2 a the $Y^{+}$distributions along the coolant surface which have not exceed 0.2 around the coolant hole. A grid independence test (Figure 2b) was performed to check the grid validity. Based on figure $2 b$, the centreline effectiveness can be converged within a fine mesh of $\approx 3$ million cells. Increasing to $\approx 4.5$ million cells shows insignificant variance. To save the computational time, a fine mesh of $\approx 3$ million cells is used to achieve the simulation.

Figure 3 shows the measured mean flow at three different streamwise locations (i.e., $X / D=0.0,3$, and 6) in comparison with the predicted velocity. Noteworthy, the measured data was performed with hotwire anemometry (Mini-CTA-54T42) with a sampling rate of $4000 \mathrm{fps}$ for $5 \mathrm{~s}$. The measured results were well predicted except the nearwall which is absent. However, the general agreements provide good confidence for the predicted flow physics.

Figure 4 shows the time-average effectiveness in comparison with the measured data. Apparently, LES results fairly agree with the experiment, though the lateral diffusion is still less significant than the experiment. This could be the contribution of the grid resolution as thought by Kim and Hassan (Kim \& Hassan, 2010), though figure 2 showed acceptable grid refinement. Some discrepancies observe in the near-hole $(1<X / D<4)$ at $M=0.8$ which might due to the flow separation effects at such high blowing ratio (Johnson et al., 2014), which lead to huge coolant instability. Furthermore, Ling (Ling et al., 2015) found an error introduced to the simulation at the near-wall region, and thus could lead to large discrepancy in effectiveness values.

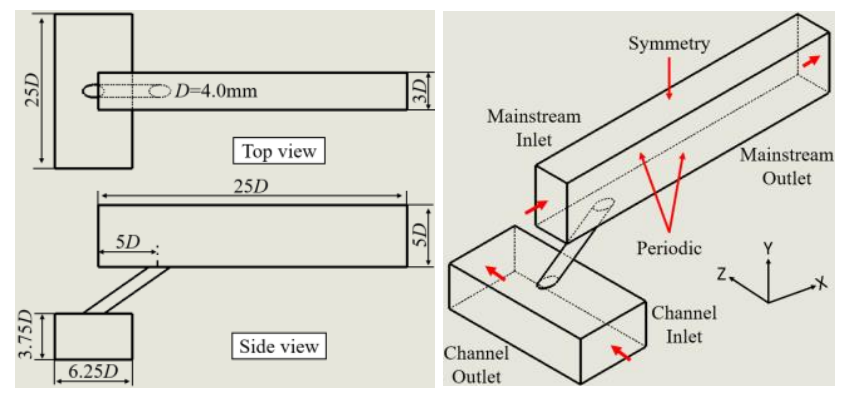

(a) Geometry

(b) Boundary conditions

Figure 1. Numerical setup

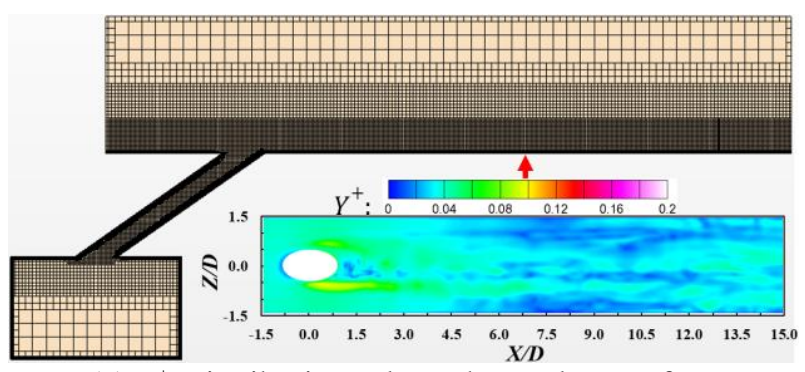

(a) $Y^{+}$Distributions along the coolant surface

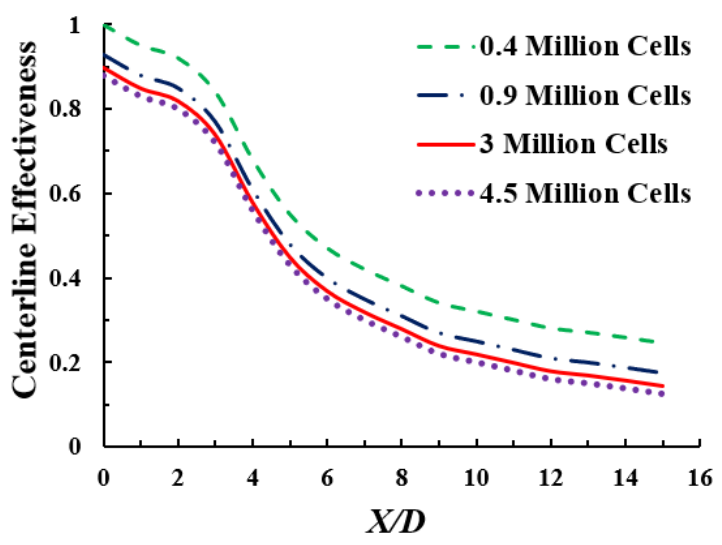

(b) Grid independent check

Figure 2. Mesh validation, $M=0.4$

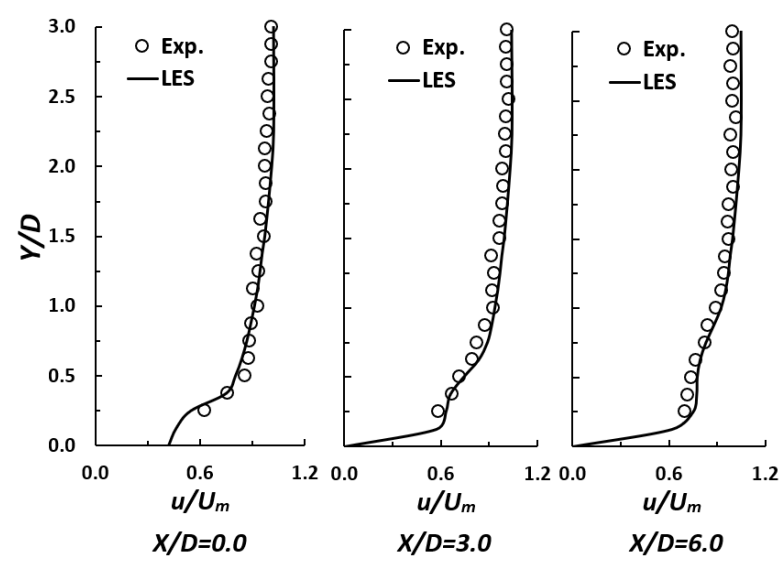

Figure 3. Mean velocity profiles at $M=0.4$ 


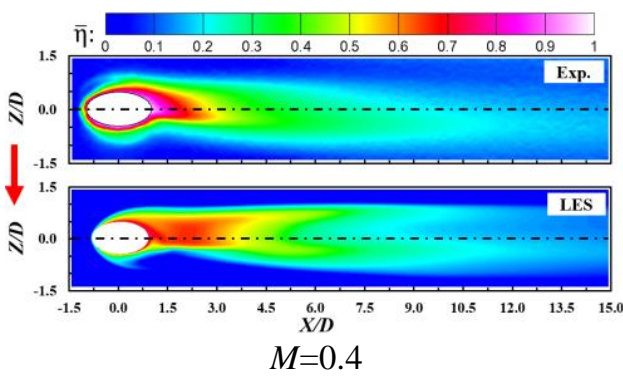

(a) Effectiveness contours

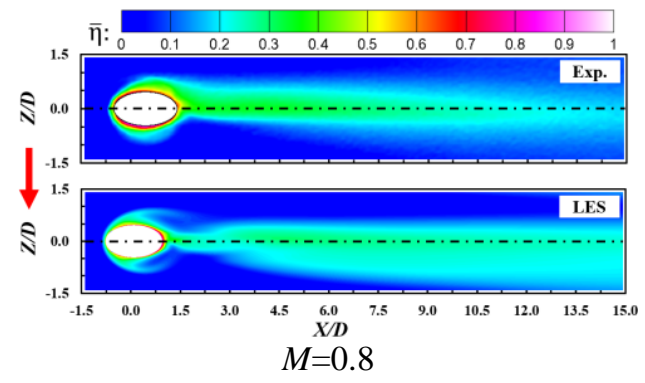

$M=0.8$

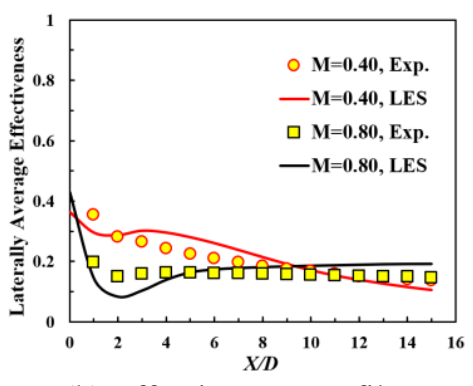

(b) Effectiveness profiles

Figure 4. Time-average film cooling effectiveness for LES validation

\section{RESULTS AND DISCUSSIONS}

\section{Time-average effectiveness and flow field}

Figure 5 shows the time-average film cooling effectiveness at both blowing ratios. The red line arrow for all figures indicates the internal flow direction. At $M=0.4$ (Figure 5a), the effectiveness is laterally spread and attached to the coolant surface. Higher effectiveness values $(\eta>0.5)$ exists in the near-hole $(X / D<3)$ and slightly decreases downstream $(3<X / D<15)$. The laterally average effectiveness profile (Figure $4 \mathrm{~b}$ ) affirms this observation, which mainly caused by insufficient momentum/coolant amount at such low blowing ratio. The effectiveness is asymmetrically distributed. It is shifted to the positive side in the near-hole $(X / D<3)$ and flaps to the negative side downstream $(X / D>3)$. This feature is clearly captured in the lateral contours at different streamwise locations (Figure 5a). The coolant is well attached to the surface at the internal flow side (hole positive side) and clearly separates upward at the opposite side (hole negative side). This is mainly because the coolant feed direction effects as reported by other researchers (Acharya \& Leedom, 2012; Peng \& Jiang, 2012). When the blowing ratio is increased to $M=0.8$, the near-hole effectiveness $(1<X / D<3)$ is suddenly decreased, resulted in flow separation (Figure 5b) due to the highly coolant momentum. The lateral profile (Figure 4b) shows sharp decay in the effectiveness values and slightly increases downstream, which indicates jet lift-off and reattachment behavior (Johnson et al., 2014). For asymmetrically distribution, the effectiveness exhibits the same behavior as $M=0.4$ (i.e., shifts to the positive side in the near-hole $(X / D<3)$ and flaps to the negative side downstream). However, the lateral contours show quite symmetric distribution at $X / D=1$, and 3 . The internal flow is directly responsible for this asymmetric effectiveness, which could reshapes itself after reattachment.

Figure 6 shows the time-average flow field along the coolant hole at different blowing ratios. At $M=0.4$, the streamlines show sudden flow movement at the back side (back and front sides refer to the downstream and upstream of the coolant hole, respectively). The coolant shifts to the front side to form a high-velocity region and leave a lowpressure region at the opposite side, which refers to the skewed vena-contracta at the hole entrance due to the flow impingement. Through $\left(S_{1}-S_{4}\right)$, the velocity distribution shows a high flow velocity at the hole periphery and lowvelocity at the hole core. Their position changes with respect to the cross-sectional position $(S)$, which indicate their rotation. As a result, minor helical flow observes at the hole periphery and major swirl flow observes at the hole core (Alekseenko et al., 1999). During their development, there is a distortion flow in-between that merge with the helical flow along the coolant hole, which could be rapid distortion problem (Hunt \& Carruthers, 1990). When the blowing ratio is increased to $M=0.8$, the high-velocity region/low-pressure region becomes larger, and the vena-contracta behaviour becomes stronger due to high coolant momentum. Significant distortion flow observes that distorts the hole boundary layer and merges with the helical flow at the hole periphery (Alekseenko et al., 1999). In accordance, the helical flow becomes major and the swirl flow becomes minor.

Noteworthy, the flow and the pressure development along the coolant hole $\left(S_{1}-S_{4}\right)$ at both blowing ratio lead to differences in the jet exit flow behavior that is responsible for the differences in the effectiveness values, and thus figure 7 shows the coolant behavior at the hole inlet/exit. At $M=0.4$, the entrance streamlines show strong swirl flow at the lowpressure region (flow circulation region) and extend along the hole core. It strongly shifts to the negative side at the hole exit, and mostly develops upward. This could explain the poor effectiveness in the near-hole negative side, and the enhancement in the vertical direction at different streamwise locations $(X / D=1,3$, and 5 in figure $5 a)$. On the other hand, the helical flow starts from the high-velocity region at the hole annular. It observes at the hole positive side with a wider lateral distribution at the hole exit and attaches the coolant surface due to the suction effects. This clearly explains the better effectiveness distribution in the near-hole positive side (Figure 5a). Similarly, the entrance streamlines at $M=0.8$ show equivalent chance of swirl and helical flows, and thus become quite equally distributed at the hole exit. The swirl flow completely lift-off the coolant surface in the near-hole $(1<X / D<3)$ and attaches downstream, whereas the helical flow quite attaches the coolant surface at the positive side. It is consistent with the effectiveness distribution in figure $5 \mathrm{~b}$ and explains its behavior. Furthermore, the flow appears symmetrically upward due to the reattachment behavior which explains the effectiveness distributions in the vertical direction at different streamwise locations (i.e., $X / D=1,3$ and 5 in figure $5 b$ ).

After all, the coolant swirl flow leads to a strong coolant mixing with the mainstream which carries it to the negative side downstream. Meanwhile, the helical flow leads to a coolant lateral spreading which carries it to the positive side in the near hole. Both of them lead to damp the jet lift-off and improve the coolant effectiveness. 


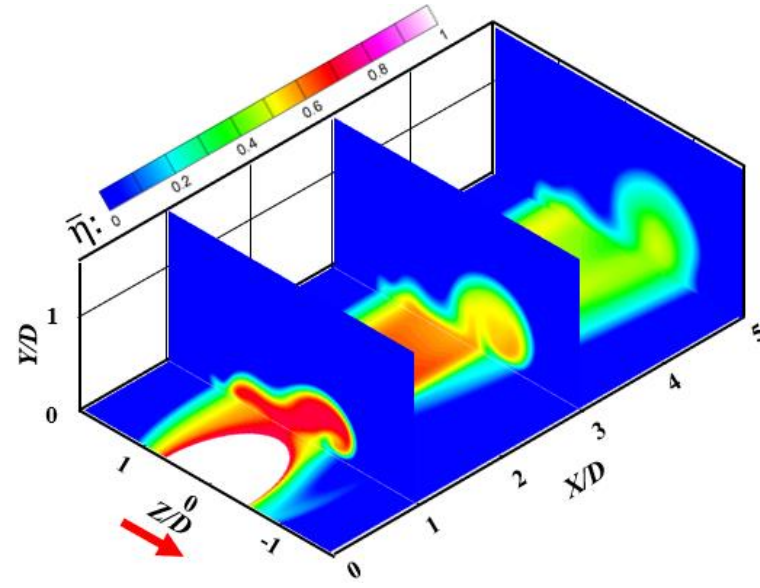

(a) $M=0.4$

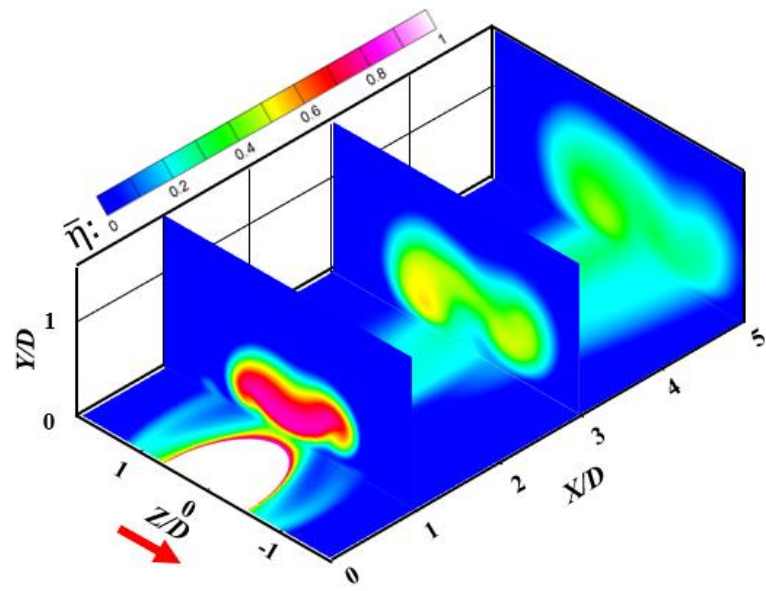

(b) $M=0.8$

Figure 5. Time-average film cooling effectiveness distributions

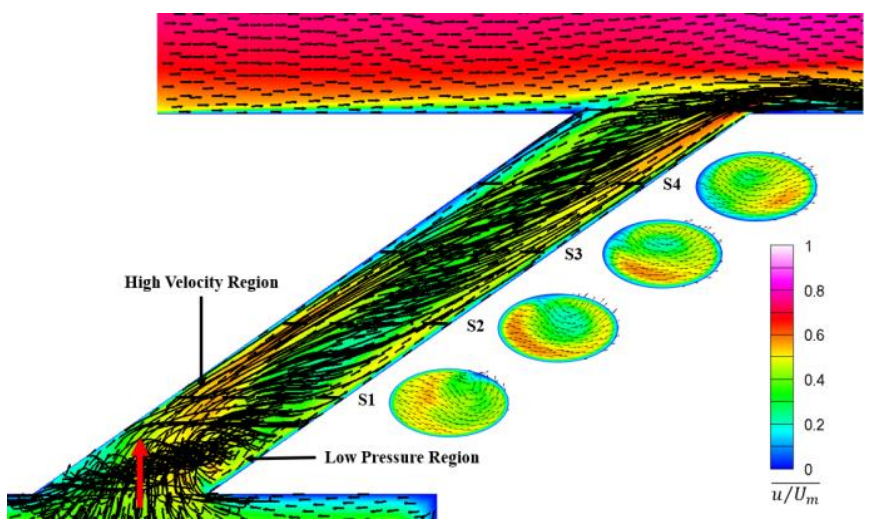

(a) $M=0.4$

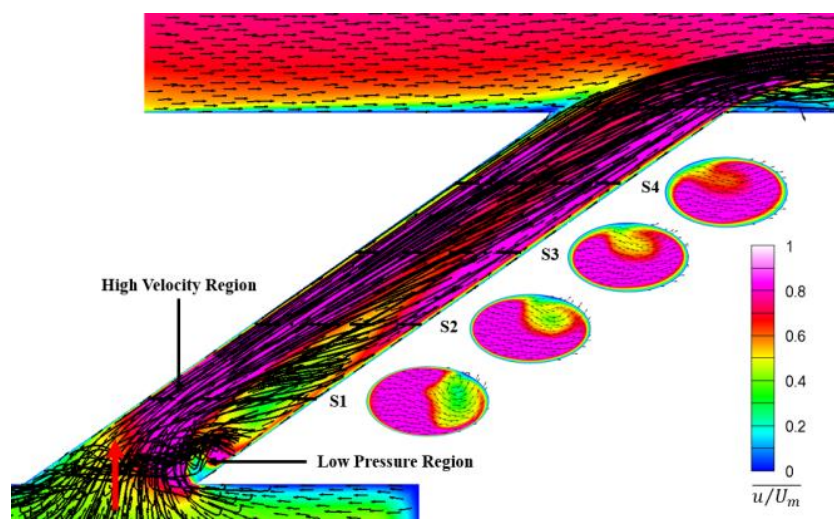

(b) $M=0.8$

Figure 6. Time-average flow field distributions along the coolant hole, $Z / D=0.0$
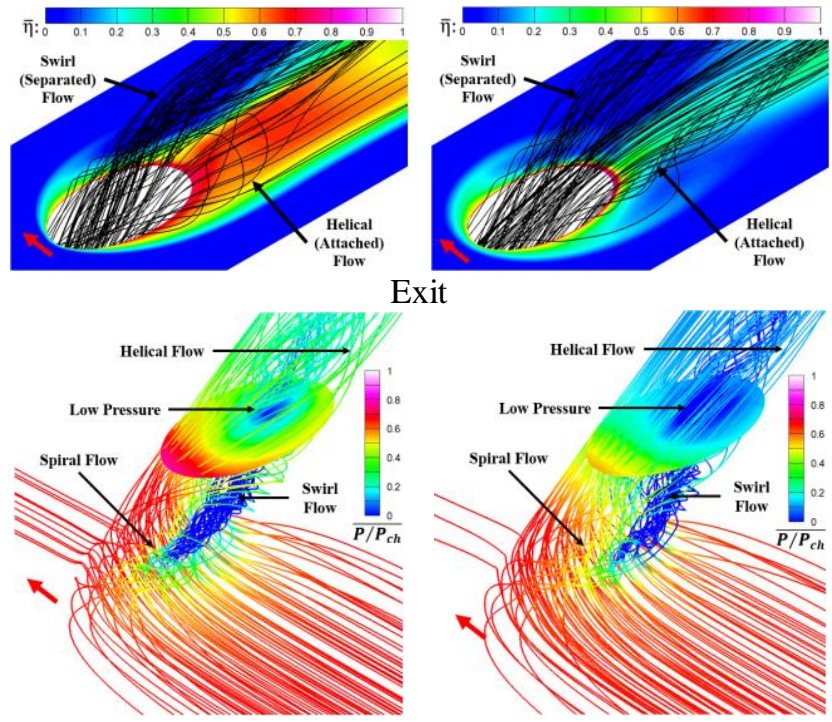

(a) $M=0.4$

(b) $M=0.8$

Figure 7. Coolant behaviour

\section{Vortical structures}

For figures $8-13$, the streamwise vorticity $\left(\omega_{x}\right)$ is used to mapped the vortex structures. Figure 8 shows the instantaneous vortical structures identified by Q-criterion isosurface (Hunt et al., 1988) which is defined as;

$$
Q=\frac{\left(\Omega_{i j} \Omega_{i j}-S_{i j} S_{i j}\right)}{2}
$$

$\Omega_{i j}$ is the rotation rate:

$$
\Omega_{i j}=1 / 2\left(\left(\partial u_{i} / \partial x_{j}\right)-\left(\partial u_{j} / \partial x_{i}\right)\right) .
$$

$S_{i j}$ is the strain rate:

$$
S_{i j}=1 / 2\left(\left(\partial u_{i} / \partial x_{j}\right)+\left(\partial u_{j} / \partial x_{i}\right)\right) .
$$

Based on Dubief and Delcayre (Dubief \& Delcayre, 2002), positive Q-isosurfaces is used to identify the coherent vortical structures because it demonstrates the structure, which its strain overcomes its rotation, and thus making those surfaces eligible as a vortical structure.

Figure 8a shows the instantaneous vortical structures at $M=0.4$. As a result of the entrance behavior, the spiral vortex is formed at the internal flow side. At low-pressure region, a strong swirl vortex (red map) is formed due to the skewed vena-contracta, which associated and consistent with the swirl flow (Figures 6a and 7a). It becomes dominant, and developed along the coolant hole (see $S_{1}-S_{4}$ in Figure 9a). In association with the helical flow, the helical vortex (blue) is formed at the hole periphery. Both (swirl and helical vortices) act as a vortex tube, which based on the previous studies (Alekseenko et al., 1999; Mitrofanova et al., 2013) can form swirl and helical vortices at the hole core and periphery, 
respectively. In accordance with the distortion flow, several distortion vortices are formed and merged with the helical vortex (Hunt \& Carruthers, 1990). At $M=0.8$ (Figure 8b), the swirl vortex becomes weaker, the distortion vortices become stronger, and the spiral vortex becomes insignificant compared by $M=0.4$. The helical vortex becomes significant and pair with the swirl vortex. This observation can be clearly seen in figure $9 \mathrm{~b}$, which caused by the high flow momentum inside the coolant hole.

Upstream the hole (Figure 8a), a horseshoe vortex is formed at the hole leading edge because the upstream boundary layer approaches the coolant jet. Its positive leg (negative hole side) is stronger than the negative leg (positive hole side). Although previous studies (i.e., Sakai et al., 2014; Zhong et al., 2016) reported the absence of the horseshoe vortex in the turbulent boundary layer, the swirl flow disturbs the mainstream boundary layer and produces the horseshoe vortex. At $M=0.8$, the swirl flow no longer disturbs the mainstream boundary layer. According, both legs are weak and quite decline as shown in figure $8 \mathrm{~b}$.

At the hole lateral edges (Figure 8a), the K-H vortex is formed as a result of the $\mathrm{K}-\mathrm{H}$ instabilities from coolant-tomainstream shearing (Andreopoulos \& Rodi, 1984; Karagozian, 2014; Mahesh, 2011). It characterises the turbulent flow and grows parallel to the mainstream flow downstream the coolant hole. Its structure is strong at the hole negative side and relatively week at the hole positive side. This observation is clearly seen from the vorticity distribution (Figure 10a) at the hole lateral edges $(Z / D= \pm 0.5)$. Based on figure $7 \mathrm{a}$, the flow behaviour is the main responsible for the strength of this structure because the positive side associated with the helical flow, and the negative side associated with the swirl flow. Consequently, the strength of the $\mathrm{K}-\mathrm{H}$ vortex at $M=0.8$ (Figure $8 \mathrm{~b}$ ), is quite equal

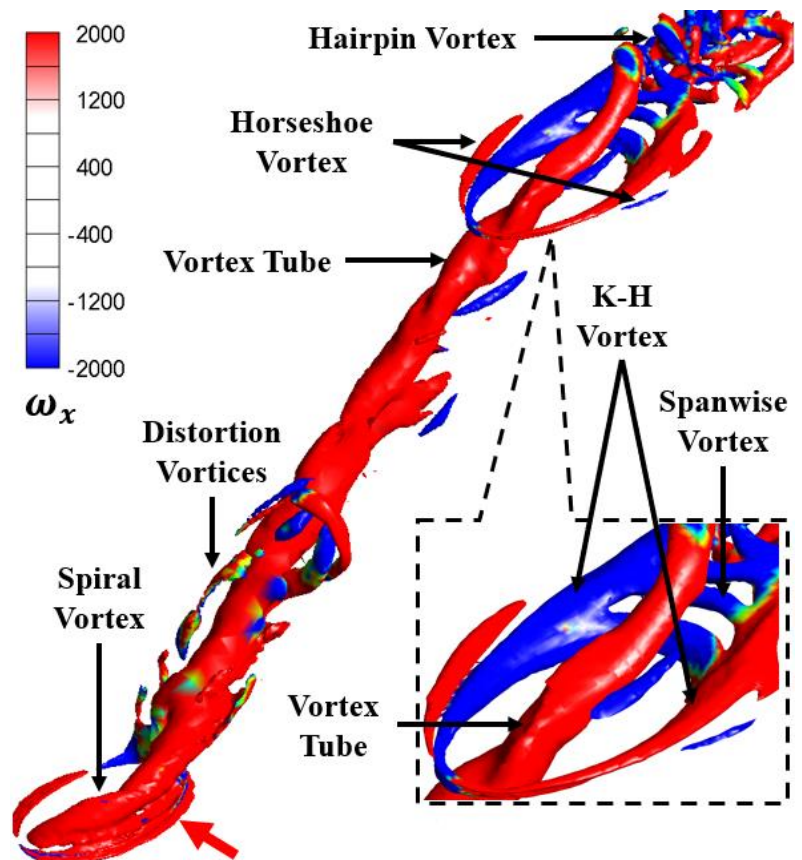

(a) $M=0.40$ strengthen as indicated by the vorticity distribution at the hole lateral edges $(Z / D= \pm 0.5)$ in figure $10 \mathrm{~b}$.

At the hole trailing edge (Figure 8a), the spanwise vortex is formed. It has a negative vorticity map that associates with the $\mathrm{K}-\mathrm{H}$ vortex at the hole lateral edges, and interacts with the mainstream flow at the hole trailing edge (Figure 10a). The flow is suctioned at the hole trailing edge (low-pressure region) between the $\mathrm{K}-\mathrm{H}$ vortex legs, and thus the spanwise vortex redistributes the boundary layer vorticity. Usually, it pulls the boundary layer vorticity away instead of creating wall vorticity (Kelso et al., 1996; Luton et al., 1995). At $M=0.8$ (Figure $8 \mathrm{~b}$ ), the spanwise vortex no longer attaches the coolant surface which reflects the coolant lift-off in the near-hole region $(1<X / D<3$ in figure $5 \mathrm{~b}$.

At the hole exit (Figure $8 \mathrm{a}$ ), the vortex tube (i.e., swirl and helical vortices) is significantly formed. It primary orient at the positive side and immediately shifts to the negative side upward the hole trailing edge. Based on figure 10a, the swirl vortex is dominant at the hole centerline $(Z / D=0.0)$ and negative side $(Z / D=-0.5)$. Its orientation deforms instantaneously into a rolling shape at the hole negative side, which swirls around the K-H negative leg downstream. At $M=0.8$ (Figure $8 \mathrm{~b}$ ), the vortex tube is less significant because highly coolant momentum. It is observed at $Z / D=0.0$ and $Z / D=-0.5$ (Figure 10b), and still deforms a quite asymmetric rolling shape that swirls around the K-H negative leg.

Figures 9 and 10 show a significant shear vortex that is formed at the hole leading edge (upward the hole exit). It cannot be observed in the vortical structures (Figure 8) due to its incoherent. It is resulted from the $\mathrm{K}-\mathrm{H}$ instabilities caused by the coolant-to-mainstream interactions (Kalghatgi \& Acharya, 2014; Mahesh, 2011). It is converted to a hairpin vortex downstream. Meanwhile, these hairpin vortices lead to coolant reattachment downstream at $M=0.8$.

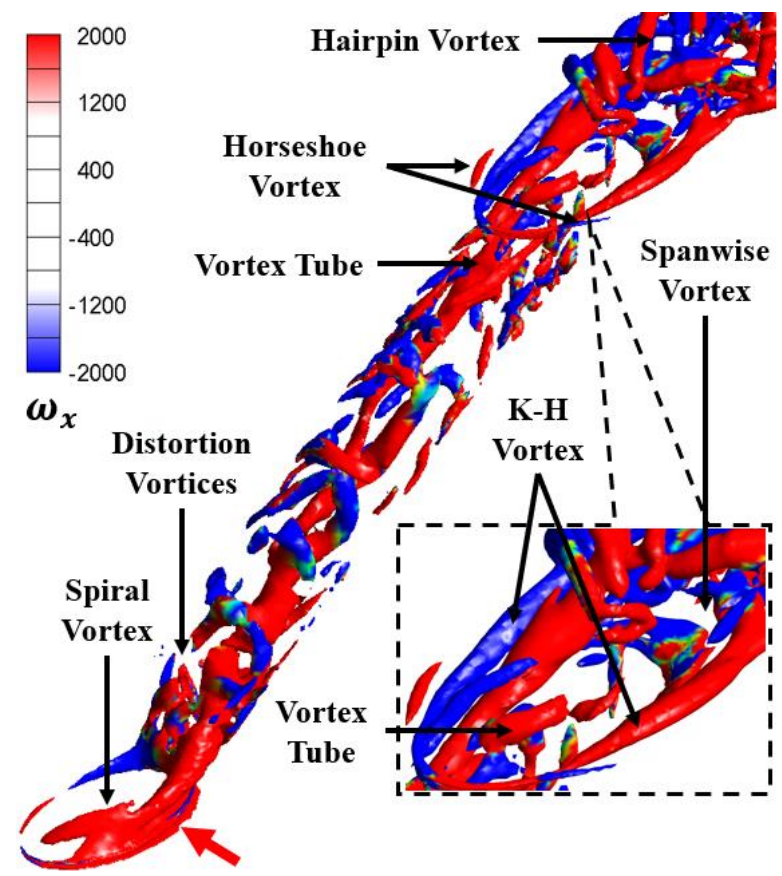

(b) $M=0.80$

Figure 8. Instantaneous vortical structures (Q- criterion isosurfaces and vorticity $\omega_{x}$ ) 


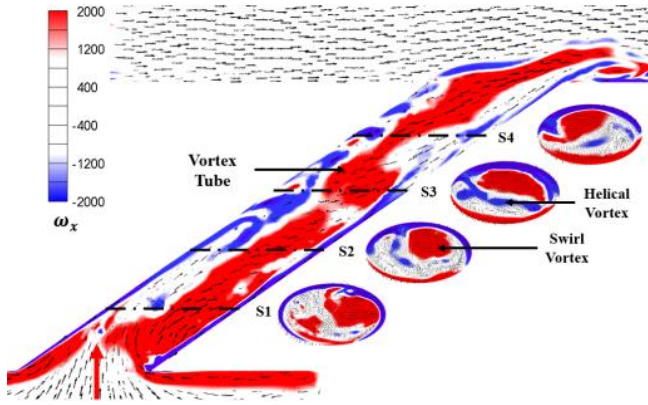

(a) $M=0.4$

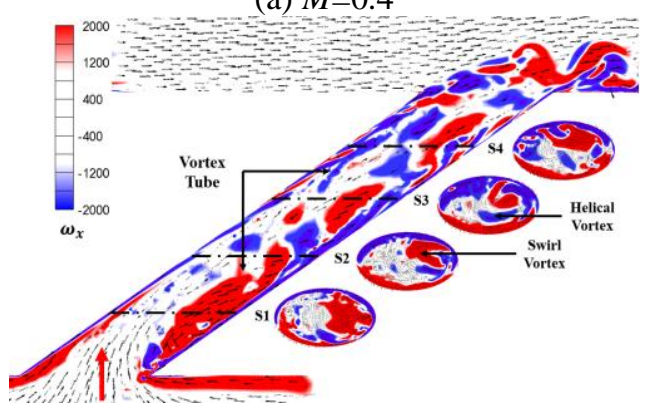

(b) $M=0.8$

Figure 9. Instantaneous flow vorticity $\omega_{x}$ along the coolant hole

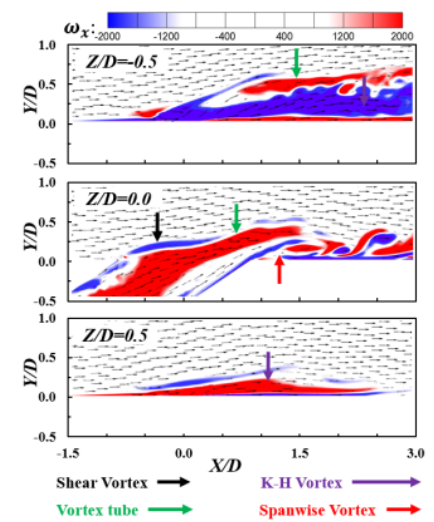

(a) $M=0.4$

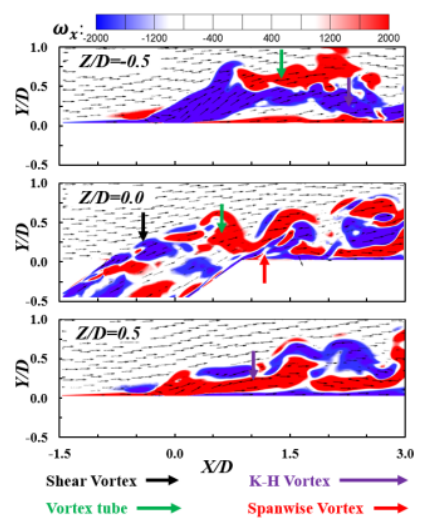

(b) $M=0.8$
Figure 10. Instantaneous flow vorticity $\omega_{x}$ at the hole exit with different $Z / D$ plans

\section{Origin of the vortical structures}

Figure 11 shows the time-average coherent vortical structures mapped by the time-average vorticity and velocity vectors/streamlines $3 D$ downstream. Figure 11a shows the vortical structures of plenum feed which reproduced from Kalghatgi and Acharya (Kalghatgi \& Acharya, 2014) for better explanation of the crossflow effects. The schematic shares approximately similar velocity ratio $(V R=0.5)$ at $M=0.4$. The structure includes horseshoe vortex (formed at the hole leading edge), CRVP from the K-H vortex (formed at the hole lateral edges), and spiral structure (formed at the hole trailing edge). The CRVP and the spiral structure merged approximately $2 D$ downstream. Kim and Hassan (Kim \& Hassan, 2010) reported similar structure.

Figure (11b) shows the vortical structures of the present study at $M=0.4$. Unlike plenum (Figure 11a), the structures orient at the hole negative side. The horseshoe vortex structure is formed at the hole leading edge with strong and narrow positive leg than the negative leg. Similarly, the K-H vortex structure is formed with strong negative leg than the positive leg at the hole lateral edges. At the hole trailing edge, the spanwise vortex is converted to spiral structure. Immediately, it is merged with the K-H vortex structure and oriented at the hole negative side. They are formed the CRVP downstream as will discussed in details in the next section. At the hole exit, the vortex tube structure (i.e., swirl and helical vortices) is significantly formed. The vortex tube (swirl vortex) is oriented at the hole negative side with coherent structure. It swirls around the CRVP negative leg and creates a rotating vortex (RV). While the helical vortex is incoherent, it interacts with the mainstream flow and forms a wall vortex (WV) downstream due to the boundary layer approach. Other researchers (Kelso et al., 1996; Schlegel et al., 2011) reported the contribution of the mainstream boundary layer to the formation of the WV.

Figure 11c shows the vortical structures of the present study at $M=0.8$. The horseshoe vortex structure is weakly formed. The K-H vortex structure is quite equally distributed. The spiral structure becomes clearer and separated from the surface which reflects the coolant lift-off. The K-H and spiral structures are merged downstream to form the CRVP. The vortex tube structure is no longer significant. Still, the structure shows the coherent of the swirl vortex, which orients at the hole negative side. It creates a RV that swirls around the $\mathrm{K}-\mathrm{H}$ negative leg. The helical vortex is incoherent and doesn't have any signature downstream.

Figure 12 shows the time-average flow vorticity $\omega_{x}$ at different streamwise locations. The time-average flow field (velocity vectors and streamlines) is used to highlight the formation of the CRVP and the signature of the vortex tube. At $M=0.4$ (Figure 12a), the time-average velocity vectors and streamlines at $X / D=0.0$ show early flow rotations at the hole lateral edges. It reflects the early formation of the CRVP and thus the contribution of the K-H vortex to its formation. The in-tube boundary vortex extends to the $\mathrm{K}-\mathrm{H}$ vortex which indicate its contribution to the CRVP (Walters \& Leylek, 1997). At $X / D=1.0$, the K-H vortex becomes the dominant and clearly forms the CRVP. The signature of the vortex tube (RV from the swirl vortex) is clearly formed at the hole negative side in-between the legs of the CRVP. It is developed with the K-H positive leg and swirls around its negative leg (see $X / D=3$ ). It forces the $\mathrm{K}-\mathrm{H}$ negative leg to move away from the geometrical centerline $(Z / D=0.0)$ downstream $(X / D=3$ and 5). On the other hand, the vortex tube (WV from the helical vortex) is developed from the wall shearing at the hole positive side $(X / D=1)$ and forms the WV downstream due to the boundary layer approach (Kelso et al., 1996; Schlegel et al., 2011). Similarly, at $M=0.8$ (Figure $13 \mathrm{~b}$ ), the K-H and the in-tube boundary vortices contributes the CRVP. At $X / D=1$, the K-H vortex is dominantly formed the CRVP. The vortex tube (RV from the swirl vortex) still observes at the hole negative while no signature for the WV from the helical vortex. Downstream $(X / D=3$ and 5), the K$\mathrm{H}$ vortex is the main responsible of the CRVP formation. Therefore, the vortex tube is the main responsible for the coolant spreading at both blowing ratios.

\section{Unsteady behaviour}

Figure 13 shows the unsteady behaviour of the coolant effectiveness due to the vortical structures. Figure 13a shows typical instantaneous effectiveness distribution over the 


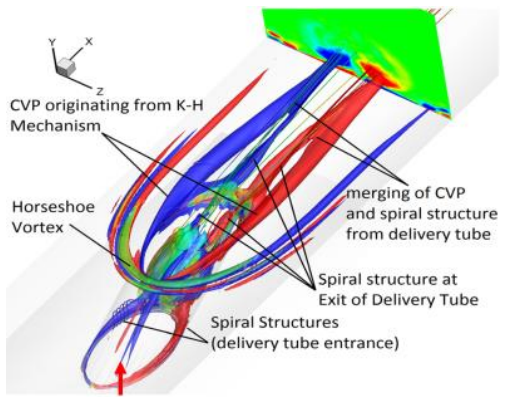

(a) $V R=0.5$, Plenum, (Kalghatgi \& Acharya, 2014)

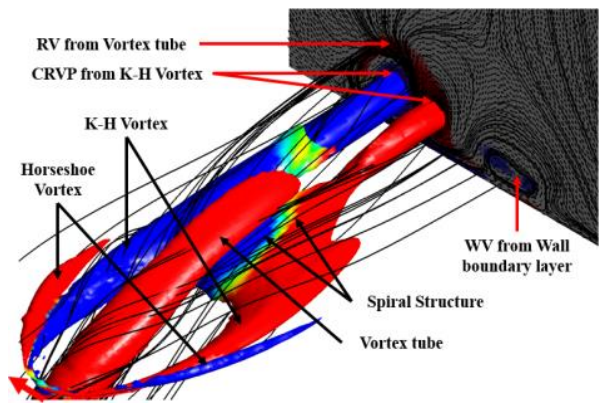

(b) $V R=0.41$, Crossflow, Present study

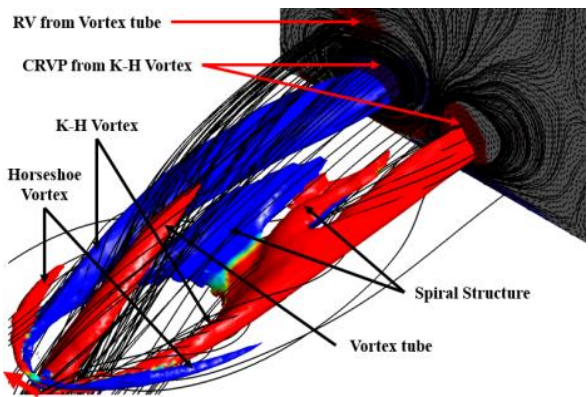

(c) $V R=0.82$, Crossflow, Present study

Figure 11. Time-average coherent vortical structures $\left(Q\right.$ - criterion isosurfaces and vorticity $\left.\omega_{x}\right)$

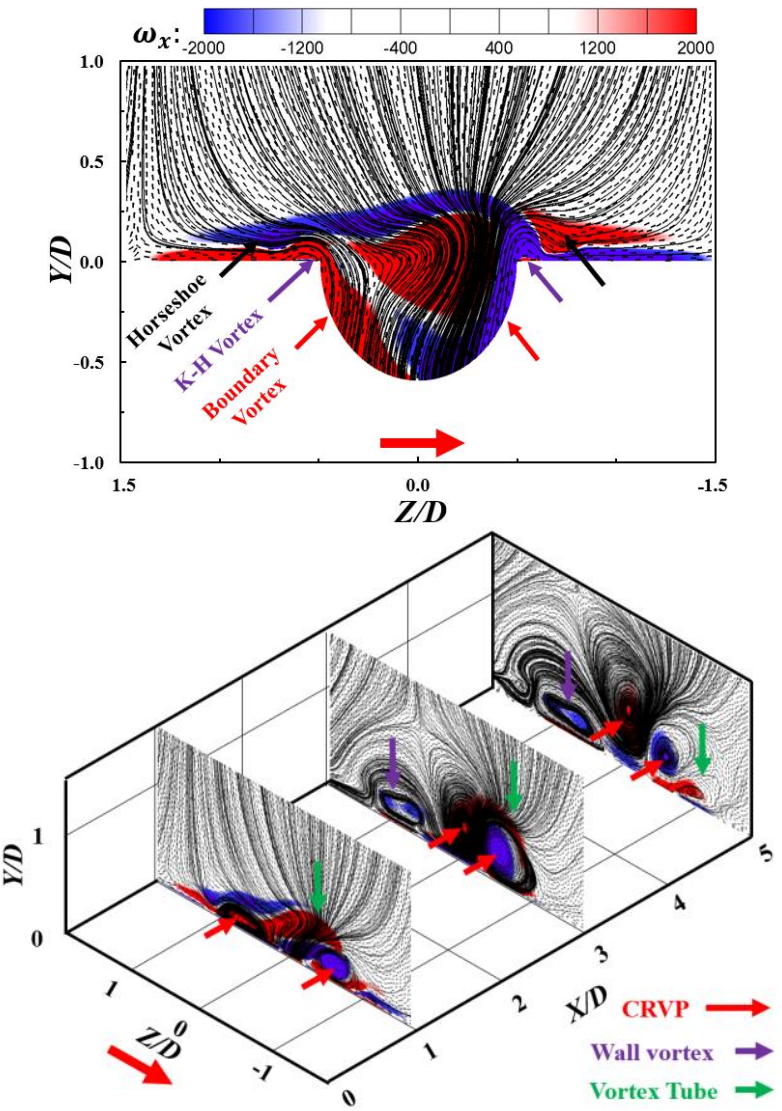

(a) $M=0.4$
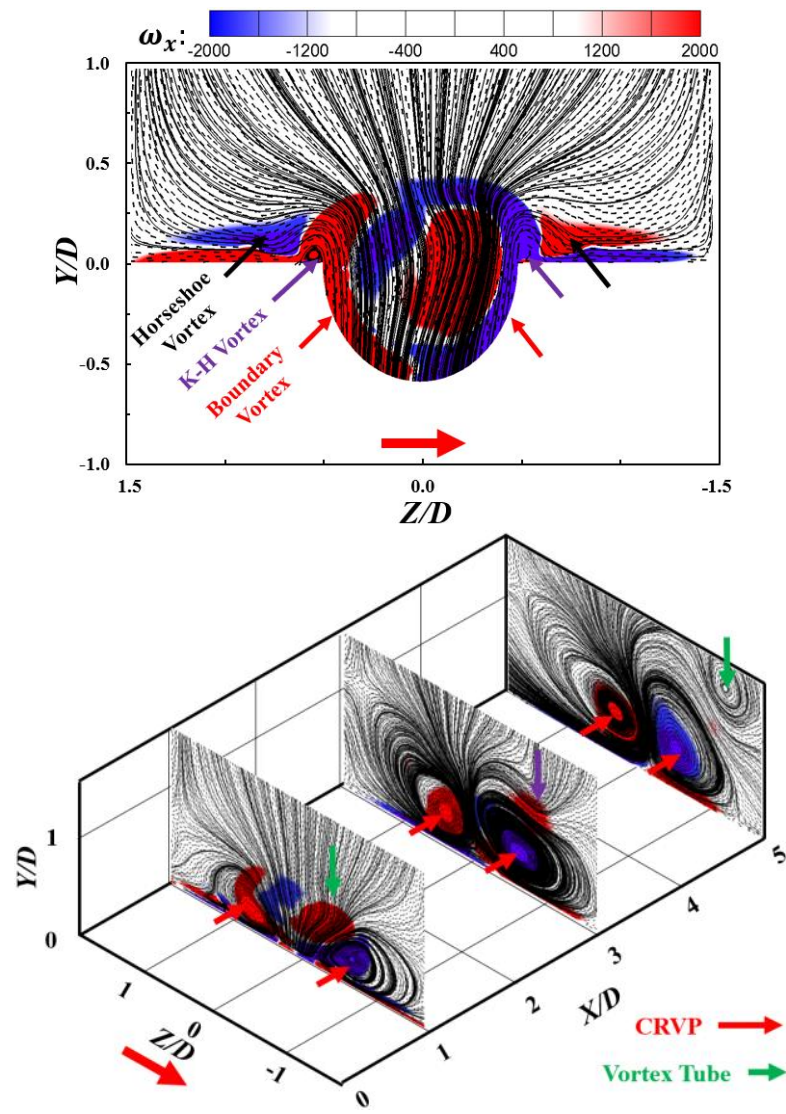

(b) $M=0.8$

Figure 12. Time-average flow vorticity $\omega_{x}$ and flow distribution at different lateral locations

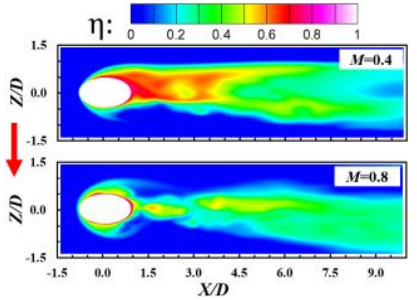

(a) Instantinouse

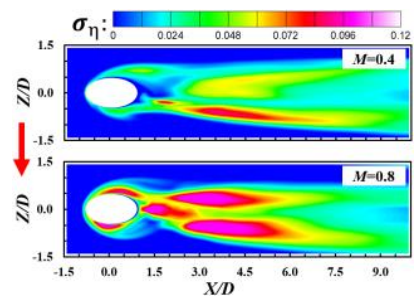

(b) Standared deviation (SD)

\section{Figure 13. Unsteadiness of the effectiveness} over the coolant surface

coolant surface. It can be clearly seen the highly unstable film cooling distribution, which mainly caused by the vortical structure (see Figure 8) due to coolant-to-mainstream interactions. The corresponding standard deviation (SD) of the instantaneous effectiveness is shown in figure 13b. It is inversely proportional to the time-average effectiveness (see

Figure 5) and reflects the coolant fluctuations over the coolant surface.

At $M=0.4$, the effectiveness is asymmetrically fluctuated around the coolant hole as indicated by the SD contour. The near-hole region $(X / D<2.5)$ shows relatively strong fluctuation at the hole positive side than the negative side. Based on the vortical structures (Figure 11b), the K-H vortex structure is the main responsible for such fluctuation because it originates primary at the hole lateral edges and slightly shifts to the hole negative side. Another possible reason could be the interactions of the K-H vortex with the horseshoe legs. The core region $(1<X / D<2.5,-0.25<Z / D<0.5)$ shows low coolant fluctuation, which reveals the stability of the spiral structure. Downstream the coolant hole $(X / D>2.5)$, highly coolant fluctuation is observed at the hole negative side. The SD shows two unstable columns (i.e., $(2.5<X / D<7)$ at the negative side and $(3.5<X / D<4.5)$ at the hole centreline). 
Based on figure $11 \mathrm{~b}$, the interactions of the CRVP structure with the vortex tube structure is the main contributor for such highly unsteadiness (see Figure 12a).

At $M=0.8$, the fluctuation in the near-hole region becomes strongly asymmetric. Recalling figure $11 \mathrm{c}$, either the K-H structure or its interactions with the horseshoe legs could be responsible for such fluctuation. At the hole core $(1<X / D<2.5)$, highly fluctuation is observed, which indicates the instability of the spiral structure due to the mainstream shear layers at the trailing edge (see Figure 10b). Downstream the coolant hole $(X / D>2.5)$, the fluctuation becomes strong (i.e., two highly unstable columns $(2.5<X / D<7)$ at the lateral edges) with wider fluctuations at the negative edge. This unsteadiness could be caused by the coolant lift-off and reattachment which regenerate the CRVP (Sakai et al., 2014). Another possible reason could be the interaction of the CRVP structure with the vortex tube structure (see Figure 12b).

\section{CONCLUSIONS}

LES was conducted to investigate the internal crossflow effects on the vortical structures of the film cooling flow. The coolant was fed by an internal channel through a circular hole at blowing ratio of ( $M=0.4$ and 0.8$)$. The vortical structures (i.e., CRVP and Vortex tube) were identified clearly by the Q-criterion coherent iso-surfaces and their fluctuation were identified by the SD. The main conclusions are:

- The internal flow was significantly affected on the coolant performance. It was asymmetrically distributed, (i.e., higher values located at the internal flow side in the near-hole and shifted to the opposite side downstream).

- The internal flow was formed swirly at the hole core and formed helically at its periphery, and both acted as a vortex tube along the coolant hole. It was formed due to the skewed vena-contracta at the hole entrance, where low-pressure region was developed. The swirl flow was developed upwind at the hole exit and declined the effectiveness, whereas the helical flow was attached to the surface and enhance the coolant effectiveness.

- The CRVP was originated from the K-H vortex (significant contribution) and the in-tube boundary vortex. A vortex tube structure was formed along the coolant hole. It became the dominant coherent structure, and was responsible for coolant-to- mainstream shearing (K-H instabilities) at the hole exit, and thus forced the $\mathrm{K}$ $\mathrm{H}$ vortex to spread downstream.

- The interaction of the CRVP and the vortex tube structures was significantly increased the coolant instability. Furthermore, the unsteadiness at $M=0.8$ was caused by the jet lift-off and reattachment behaviour.

- Noteworthy, this work demonstrates the vortical structures due to the internal flow effects, which significantly affected by the channel velocity ratio $\left(V R_{c h}\right)$. However, only $V R_{c h}=0.4$ was investigated, and thus further investigation of $V R_{c h}$ might be needed.

$\begin{array}{ll}\text { NOMENCLATURE } \\ C R V P & \text { Counter-rotating vortex pair } \\ D & \text { Hole diameter } \\ D R & \text { Density ratio } \\ J I C F & \text { Jet in cross-flow }\end{array}$

$\begin{array}{ll}K-H & \text { Kelvin Helmholtz } \\ L E S & \text { Large eddy simulation } \\ M & \text { Blowing ratio } \\ P & \text { Instantaneous pressure } \\ \bar{P} & \text { Time-average pressure } \\ R e & \text { Reynold number } \\ R V & \text { Rotating vortex } \\ S D & \text { Standard deviation } \\ T & \text { Temperature } \\ U & \text { Velocity } \\ V R & \text { Velocity ratio } \\ W V & \text { Wall vortex } \\ \text { Greek } & \\ \delta & \text { Boundary layer thickness } \\ \eta & \text { Instantaneous adiabatic cooling effectiveness } \\ \bar{\eta} & \text { Average adiabatic cooling effectiveness } \\ \sigma_{\eta} & \text { Effectiveness SD } \\ \mu & \text { Dynamic viscosity } \\ \rho & \text { Density } \\ \omega_{x} & \text { Streamwise vorticity } \\ \end{array}$

\section{Subscript}

$\begin{array}{ll}a w & \text { Adiabatic wall } \\ c & \text { Coolant } \\ c h & \text { Coolant channel } \\ m & \text { Mainstream } \\ i, j, k & \text { Normal vectors }\end{array}$

\section{ACKNOWLEDGMENTS}

The authors gratefully acknowledge the financial support for this study from the National Natural Science Foundation of China (11725209, 51806138) and the Shanghai Sailing Program (18YF1411300).

\section{REFERENCES}

Acharya, S., \& Houston Leedom, D. (2012). Large Eddy Simulations of Discrete Hole Film Cooling With Plenum Inflow Orientation Effects. Journal of Heat Transfer, 135(1), https://doi.org/10.1115/1.4007667

Acharya, S., \& Kanani, Y. (2017). Advances in Film Cooling Heat Transfer. In Advances in Heat Transfer (1st ed., Vol. 49). https://doi.org/10.1016/bs.aiht.2017.10.001

Alekseenko, S. V., Kuibin, P. A., Okulov, V. L., \& Shtork, S. I. (1999). Helical vortices in swirl flow. Journal of Fluid Mechanics, 382, 195-243. https://doi.org/10.1017/S0022112098003772

Andreopoulos, J., \& Rodi, W. (1984). Experimental investigation of jets in a crossflow. Journal of Fluid Mechanics, 138, 93-127. https://doi.org/10.1017/S0022112084000057

Dai, C., Jia, L., Zhang, J., Shu, Z., \& Mi, J. (2016). On the flow structure of an inclined jet in crossflow at low velocity ratios. International Journal of Heat and Fluid Flow, 58, 11-18. https://doi.org/10.1016/j.ijheatfluidflow.2015.12.001

Dubief $\uparrow$, Y., \& Delcayre $\ddagger$, F. (2002). On coherent-vortex identification in turbulence. Journal of Turbulence, 1 , N11. https://doi.org/10.1088/1468-5248/1/1/011

Eberly, M. K., \& Thole, K. A. (2013). Time-Resolved Film- 
Cooling Flows at High and Low Density Ratios. Journal of Turbomachinery, 136(6), 061003. https://doi.org/10.1115/1.4025574

Fric, T. F., \& Roshko, A. (1994). Vortical Structure in the Wake of a Transverse Jet. Journal of Fluid Mechanics, 279 , $1-47$. https://doi.org/10.1017/S0022112094003800

Germano, M., Piomelli, U., Moin, P., \& Cabot, W. H. (1991). A dynamic subgrid-scale eddy viscosity model. Physics of Fluids A, 3(7), 1760-1765. https://doi.org/10.1063/1.857955

Gritsch, M., Schulz, A., \& Wittig, S. (2003). Effect of Internal Coolant Crossflow on the Effectiveness of Shaped Film-Cooling Holes. Journal of Turbomachinery, 125(3), 547. https://doi.org/10.1115/1.1580523

Hunt', J. C. R., Wray2, A. A., \& Moin3, P. (n.d.). N89-24555 Eddies, Streams, and Convergence Zones in Turbulent Flows. Retrieved from https://ntrs.nasa.gov/search.jsp?R=19890015184

Hunt, J. C. R., \& Carruthers, D. J. (1990). Rapid distortion theory and the 'problems' of turbulence. Journal of Fluid Mechanics, 212(1), 497. https://doi.org/10.1017/S0022112090002075

Johnson, B., Tian, W., Zhang, K., \& Hu, H. (2014). An experimental study of density ratio effects on the film cooling injection from discrete holes by using PIV and PSP techniques. International Journal of Heat and Mass Transfer, 76, 337-349. https://doi.org/10.1016/j.ijheatmasstransfer.2014.04.0 28

Kalghatgi, P., \& Acharya, S. (2014). Modal Analysis of Inclined Film Cooling Jet Flow. Journal of Turbomachinery, 136(8), 081007. https://doi.org/10.1115/1.4026374

Karagozian, A. R. (2014). The jet in crossflow. Physics of Fluids, 26(10). https://doi.org/10.1063/1.4895900

Kelso, R. M., Lim, T. T., \& Perry, A. E. (1996). An experimental study of round jets in cross-flow. Journal of Fluid Mechanics, 306, 111-144. https://doi.org/10.1017/S0022112096001255

Kim, S. I., \& Hassan, I. (2010). Unsteady Simulations of a Film Cooling Flow from an Inclined Cylindrical Jet. Journal of Thermophysics and Heat Transfer, 24(1), 145-156. https://doi.org/10.2514/1.33167

Kohli, A., \& Bogard, D. G. (2011). Fluctuating Thermal Field in the Near-Hole Region for Film Cooling Flows. Journal of Turbomachinery, 120(1), 86. https://doi.org/10.1115/1.2841393

Lilly, D. K. (1992). A proposed modification of the Germano subgrid-scale closure method. Physics of Fluids A, 4(3), 633-635. https://doi.org/10.1063/1.858280

Ling, J., Ryan, K. J., Bodart, J., \& Eaton, J. K. (2015). Analysis of Turbulent Scalar Flux Models for a Discrete Hole Film Cooling Flow. Journal of Turbomachinery, $\quad 138(1), \quad 011006$. https://doi.org/10.1115/1.4031698

Luton, A., Ragab, S., \& Telionis, D. (1995). Interaction of spanwise vortices with a boundary layer. Physics of Fluids, 7(11), 2757-2765. https://doi.org/10.1063/1.868654

Mahesh, K. (2011). The Interaction of Jets with Crossflow. Annual Review of Fluid Mechanics, 45(1), 379-407. https://doi.org/10.1146/annurev-fluid-120710-101115
Mitrofanova, O. V., Podzorov, G. D., \& Pozdeeva, I. G. (2013). Vortex structure of swirl flows. International Journal of Heat and Mass Transfer, 65, 225-234. https://doi.org/10.1016/j.ijheatmasstransfer.2013.06.0 07

Peng, W., \& Jiang, P. X. (2012). Experimental and numerical study of film cooling with internal coolant cross-flow effects. Experimental Heat Transfer, 25(4), 282-300. https://doi.org/10.1080/08916152.2011.609630

Peng, Wei, \& Jiang, P.-X. (2012). Large-Eddy Simulation of Film Cooling with Internal Coolant Crossflow Effects. Journal of Propulsion and Power, 28(1), 160-169. https://doi.org/10.2514/1.57115

Qenawy, M., Zhou, W., Chen, H., Shao, H., Peng, D., \& Liu, Y. (2019). UNSTEADY ANALYSIS OF ADIABATIC FILM COOLING EFFECTIVENESS BEHIND A ROW OF CIRCULAR HOLES FED BY INTERNAL CROSSFLOW. ASME Paper No GT2019-90349.

Sakai, E., Takahashi, T., \& Watanabe, H. (2014). Large-eddy simulation of an inclined round jet issuing into a crossflow. International Journal of Heat and Mass Transfer, 69, 300-311. https://doi.org/10.1016/j.ijheatmasstransfer.2013.10.0 27

Saumweber, C., \& Schulz, A. (2009, August 5). Comparison the Cooling Performance of Cylindrical and FanShaped Cooling Holes With Special Emphasis on the Effect of Internal Coolant Cross-Flow. 893-903. https://doi.org/10.1115/gt2008-51036

Schlegel, F., Wee, D., Marzouk, Y. M., \& Ghoniem, A. F. (2011). Contributions of the wall boundary layer to the formation of the counter-rotating vortex pair in transverse jets. Journal of Fluid Mechanics, 676, 461490. https://doi.org/10.1017/jfm.2011.59

Walters, D. K., \& Leylek, J. H. (1997). A Detailed Analysis of Film-Cooling Physics: Part I - Streamwise Injection With Cylindrical Holes. Volume 3: Heat Transfer; Electric Power; Industrial and Cogeneration. https://doi.org/10.1115/97-GT-269

Zhong, L., Zhou, C., \& Chen, S. (2016). Effects of approaching main flow boundary layer on flow and cooling performance of an inclined jet in cross flow. International Journal of Heat and Mass Transfer, 103, 572-581. https://doi.org/10.1016/j.ijheatmasstransfer.2016.07.0 89 